Manuelle Medizin 2017-55:3-4

DOI 10.1007/s00337-016-0226-4

Online publiziert: 31. Januar 2017

(c) Springer Medizin Verlag Berlin 2017

CrossMark
H. Tilscher

Geriatriezentrum am Wienerwald, Österreichische Ärztegesellschaft für Manuelle Medizin und konservative Orthopädie, Verein zur Prävention von Wirbelsäulenstörungen, Wien, Österreich

\title{
Knie- und Schulterschmerzen - ein häufiges Problem in der Praxis
}

\author{
Möglichkeiten und Grenzen der \\ konservativen Therapie
}

Alljährlich veranstaltet die Österreichische Ärztegesellschaft für Manuelle Medizin - konservative Orthopädie in Pörtschach am Wörthersee praxisnahe Kongresse, die sich mit den Störungen des Stütz- und Bewegungsapparates auseinandersetzen. Im Jahr 2016 waren es die Knieprobleme, die nach der Wirbelsäule die zweithäufigste Erkrankungsgruppe des lokomotorischen Systems sind. Entsprechend den epidemiologischen Erkenntnissen wurde auch auf arthritisch-arthrotische Veränderungen der Kniegelenke, speziell von älteren Frauen, hingewiesen und es wurden sowohl diagnostische als auch therapeutische, operative oder konservative Strategien diskutiert. Im Rahmen dieses Kongresses wurden aber auch Schultererkrankungen „beleuchtet“.

Im Ausbildungsschema der Österreichischen Ärztegesellschaft für Manuelle Medizin und konservative Orthopädie werden Kurse für die Untersuchung und Behandlung von Extremitätengelenken angeboten, welche die Unverzichtbarkeit der klinischen manuellen Diagnostik einmal mehr unterstreichen. So gilt auch bei morphologischen Auffälligkeiten die pathogenetische Führungsstruktur zu suchen und zu finden.

Durch die anatomisch funktionellen Gegebenheiten sind die manualtherapeutischen Zugänge an Knie- und Schultergelenk different. Das Vorliegen von so-

Kongress in Pörtschach von 08. bis 10. Juli 2016 genannten Blockierungen [1] dieser Gelenke ist eher selten/unwahrscheinlich, ein eingeschränktes Gelenkspiel oder ein vermehrtes Gelenkspiel (Instabilität) dagegen finden wir häufig. Diese beiden artikulären Dysfunktionen erfordern von uns Manualmediziner(innen) entsprechende Techniken.

Im Unterschied zum bandgesicherten Kniegelenk ergeben sich beim muskelgesicherten Schultergelenk eine Fülle von Therapiemöglichkeiten über die Muskulatur. Nach den strukturanalytischen und aktualitätsdiagnostischen Aspekten ist eine manuelle Monotherapie nicht die Regel. Neben den medikamentösen Behandlungsformen sind es die verschiedenen "Reflextherapien" (therapeutischer Reizabbau, therapeutische Reizsetzung), die in ihrer Kombination den Übergang zur Rehabilitation (Beschwerdelinderung, Beschwerdevermeidung) ermöglichen. Von besonderer Wichtigkeit ist die Prävention, bei welcher schädigende Einflüsse, auch durch Sport, diskutiert werden sollten.

\section{Die topische Diagnose „Knieschmerz"}

Die Störpotenziale der Kniegelenke hängen v. a. von Strukturen mit entsprechenden Schmerzrezeptoren ab. Bekanntlich sind Knorpel wie auch die Menisci weitgehend nozizeptorenfrei. Der Knieschmerz ist Symptom von diversen Knieerkrankungen, soll aber auch an andere Störungen denken lassen. Wie etwa in der Jugendzeit an den Morbus Perthes, später an die Coxarthrosen, die durch ihre Innervation von L3 und L4 entsprechende Projektionsschmerzen in das Knie verursachen. Ähnliches gilt auch für die radikuläre Läsion L3/L4 als Differenzialdiagnose.

\section{Die topische Diagnose "Schulterschmerz"}

Die topische Diagnose „Schulterschmerz“ - primär Ausdruck einer lokalen Erkrankung - erfordert die Berücksichtigung des übertragenen Schmerzes („referred pain") sowie der pseudoradikulären Symptomatik bei Störungen der Halswirbelsäule, aber auch bei Läsionen des Versorgungsbereiches im Plexus cervicobrachialis. Durch die Muskelsicherung

\footnotetext{
Infobox 1 Reaktive Arthritis

- Gruppe III - Diese Gruppe beinhaltet reaktive Arthritiden (ReA), deren Infektionsherd im Urogenital- bzw. Gastrointestinaltrakt liegt und entzündliche Gelenkerkrankungen verursacht. Eine Infektion im Gelenk selbst kann aber nicht nachgewiesen werden.

- Gruppe IV - Diese Gruppe beinhaltet entzündliche Arthritiden, die durch Mikroorganismen verursacht werden, bei den aber weder die Mikroorganismen selbst noch deren Produkte oder Antigene im Gelenk nachgewiesen werden können.
} 
Hier steht eine Anzeige. Springer

\section{Nichtinfizierte Arthritis}
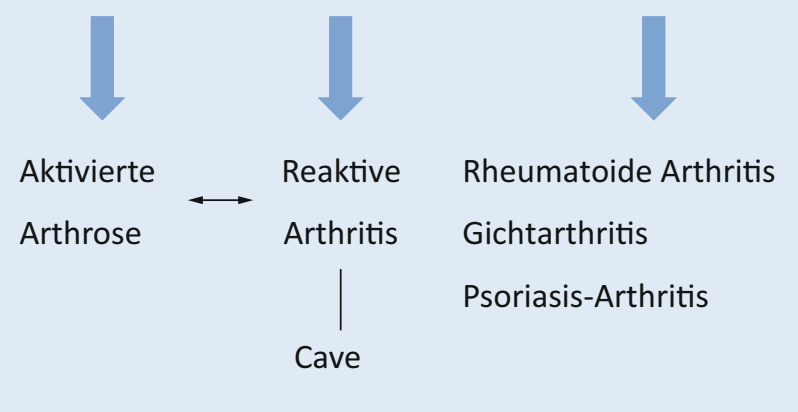

Abb. $1 \triangleleft$ Nichtinfizierte Arthritis des Schultergelenks können verschiedene Noxen in dessen Innervationsbereich eine Änderung des Bewegungsmusters, v. a. der Rotatorenmanschette bewirken, die Beschwerden verursachen, welche durch klinische Untersuchungen zu entsprechenden Eingliederungen und Therapiestrategien führen.

Zusammenfassend können nach der topischen Diagnose die sogenannten strukturanalytischen Maßnahmen der Manuellen Medizin gemeinsam mit den bildgebenden Verfahren und anderen Zusatzbefunden das Beschwerdebild definieren. Da im Vergleich zu Wirbelsäulenproblemen die Häufigkeit von sogenannten unspezifischen Beschwerden zu hinterfragen ist, könnte die Manuelle Therapie allein hinsichtlich ihres Erfolges spekulativ und von der Behandlung, ihrer Dauer und der Intensität der Techniken abhängig sein.

Wie bei allen Erkrankungen des Stützund Bewegungsapparates sind präventive, sekundärpräventive und tertiärpräventive Maßnahmen von außerordentlicher Bedeutung. Besonders die häufigen "Gonarthrosen“ bedürfen entsprechender Aktivitäten, um die lebensnotwendige Mobilität zu erhalten. So folgt die Häufigkeit der Problematik der Kniegelenke den Wirbelsäulenerkrankungen. $80 \%$ aller Menschen über 50 Jahre leiden an Gonarthrosen, auch an den sogenannten stummen Arthrosen, also Veränderungen in den bildgebenden Verfahren, die als Arthrose bezeichnet werden und keine Beschwerden verursachen [3].

Von differenzialdiagnostischer Bedeutung sind die entzündlichen Knie- gelenkerkrankungen $[2,3]$, bei welchen die aktivierten Arthrosen, d.h. die mit entzündlichen Erscheinungen auftretenden degenerativen Veränderungen, den reaktiven Arthritiden gegenüberzustellen sind, die sich im Rahmen oder im Anschluss an andere entzündliche Erkrankungen manifestieren können (• Abb. 1; • Infobox 1).

\section{Korrespondenzadresse}

\section{Prof. Dr. H. Tilscher}

Geriatriezentrum am Wienerwald,

Österreichische Ärztegesellschaft für Manuelle Medizin und konservative Orthopädie, Verein zur Prävention von Wirbelsäulenstörungen Jagdschlossgasse 59, 1130 Wien, Österreich hans.tilscher@extern.wienkav.at

Interessenkonflikt. H. Tilscher gibt an, dass kein Interessenkonflikt besteht.

\section{Literatur}

1. Moll H et al (2010) Die reversible hypomobile artikuäre Dysfunktion - die Blockierung. Man Medizin Bd 48(6):426-434

2. Zlabinger GJ (2001) Immunologische Grundlagen von Entzündungsreaktionen. In: Thumb N (Hrsg) Praktische Rheumatologie. Springer, Berlin Heidelberg, S18-41

3. Zoike E et al (2010) BKK Gesundheitsreport 2010, Gesundheit in einer älter werdenden Gesellschaft - Statistik und Analyse. Schroers-Druck GmbH, Essen 